\title{
Dipole-like fields for stabilization of systems with Pfaffian constraints
}

\author{
Dimitra Panagou, Herbert G. Tanner and Kostas J. Kyriakopoulos
}

\begin{abstract}
This paper introduces a framework that guides the design of stabilizing feedback control laws for systems with Pfaffian constraints. A new class of $N$-dimensional vector fields, the dipole-like vector fields is proposed, inspired by the form of the flow lines of the electric point dipole. A general connection between the dipole-like field and the Pfaffian constraints of catastatic nonholonomic systems is exploited, to establish systematic guidelines on the design of stabilizing control laws. The methodology is applied to the stabilization of the unicycle and of the nonholonomic double integrator. Based on these guidelines, switching control laws are constructed. The efficacy of the methodology is demonstrated through simulation results.
\end{abstract}

\section{INTRODUCTION}

Nonholonomic systems model a wide class of mechanical systems, including mobile robots, underactuated robotic manipulators, unmanned vehicles (aerial-UAVs, underwaterUUVs, ground-UGVs), surface vessels and spacecraft. Brockett's theorem [1] states that nonholonomic systems can not be stabilized by continuously differentiable, timeinvariant state feedback control laws. In fact, nonholonomic mechanical systems can not be asymptotically stabilized to a single equilibrium using any control method that employs smooth, or even continuous, time-invariant feedback [2]. Various solutions have been proposed, usually classified as piecewise continuous feedback [2], [3], time-varying feedback [4]-[8], discontinuous feedback [9]-[11] and hybrid/switching control strategies [12], [13].

The majority of these approaches, [4]-[7], [10], [11], provide stabilizing controllers for drift-free, kinematic nonholonomic systems, which are either in chained or in power form. The so-called dynamic nonholonomic systems arise either from the dynamic extension of nonholonomic kinematic systems [9], or from the modeling of underactuated mechanical systems, which induces second-order nonholonomic constraints. The controllability and stabilizability properties of these systems are addressed in [14]. A robust control architecture for mechanical systems that are subject to $p$ nonholonomic Pfaffian constraints is given in [15], which guarantees the convergence of the system to a $p$ dimensional desired manifold. The stabilization of dynamic nonholonomic systems is also addressed in [2], [8], [16][18]. Finally, various stabilization control schemes have

This work was supported by the ARL under MAST CTA \# W911NF-082-0004, through a subcontract from the University of New Mexico. Dimitra Panagou has been a visiting scholar at the Department of Mechanical Engineering at the University of Delaware. Both she and Kostas Kyriakopoulos are with the Department of Mechanical Engineering at the National Technical University of Athens. Herbert Tanner is with the Department of Mechanical Engineering at the University of Delaware. been proposed for specific systems, like the unicycle and Brockett's nonholonomic double integrator [19]-[22].

When the nonholonomic system is to be stabilized in the presence of state constraints (read obstacles), then one is faced with a nonholonomic motion planning problem. Among the variety of motion planning methods [23], potential fields [24] in general, and navigation functions (NF) [25] in particular, are popular methods since they provide closedloop feedback control laws. However, using potential fields for nonholonomic navigation brings challenges, since these systems are not capable of moving at any direction the field might require them to. The past decade has seen increased interest in the use of scalar and vector potential fields for the nonholonomic motion planning problem. A first approach is given in [26], where the discrete gradient of a Random Path Planner potential field is modified to yield a path that is consistent with the admissible directions of motion. Local minima cannot be avoided. A dipolar NF is introduced in [27] for the nonholonomic motion planning of a mobile manipulator. The idea is that its flow lines guide a unicyclelike robot to the equilibrium without explicitly stabilizing the orientation. Since its introduction, the dipolar NF framework has been extended to the motion planning of multiple unicycle-like agents in 2D and 3D environments [28]-[30]. A hybrid control scheme that incorporates the classic NF is proposed in [31] for the navigation of a unicycle-like robot subject to holonomic (sensing) constraints. In [31] the system converges to an arbitrarily small neighborhood of the goal configuration by switching between two distinct vector fields; one that is potential-decreasing and one that is potential-conserving. A harmonic potential field (HPF)based algorithm for the motion planning of a unicycle-like robot has also been proposed in [32]; this approach is similar in spirit to [27] in the sense of alignment to the field's flow lines, however the unicycle's orientation is not stabilized. For additional interesting vector field-based navigation schemes for nonholonomic robots, one may refer to [33]-[35].

This paper is inspired by [27] and proposes a more systematic control design framework, extended to a wider class of systems with nonholonomic Pfaffian constraints. A new class of vector fields, the dipole-like vector fields, is introduced. These fields are defined in $N$-dimensional vector spaces and can describe a wider class of nonholonomic systems, which includes but is not limited to the unicycle. The conceptual link between the dipole-like fields and the Pfaffian constraints of nonholonomic systems is exploited to establish a systematic method for the design of reference vector fields for nonholonomic navigation. The dimension $N$ of the fields is found to depend on the nonholonomic 
constraints. Control design guidelines are proposed and application examples are given. The perceived advantage of the proposed method is that the flow lines define a foliation of submanifolds along which the system can flow to reach the equilibrium, controlled in a reduced dimensional space. Steering the system on this foliation is an output tracking problem, which may be easier than the original nonholonomic stabilization problem, especially when state constraints are imposed. Finally, it should be noted that the 2-dimensional dipole-like field in the case of the unicycle does not coincide with the gradient of dipolar NF as they have appeared in literature, nor does its construction require any artificial obstacles.

The remaining of the paper is organized as follows: Section II provides a brief introduction on Pfaffian constraints. Section III describes the electric point dipole and the proposed dipole-like vector field. In Section IV the connection between the nonholonomic Pfaffian constraints and the dipole-like field is presented and switching control laws for the stabilization of the unicycle and of the nonholonomic double integrator are proposed. Conclusions and thoughts on further work are summarized in Section V.

\section{Pfaffian Constraints}

Consider the class of mechanical systems described by

$$
\dot{\boldsymbol{q}}=\boldsymbol{f}(\boldsymbol{q}, \boldsymbol{u}),
$$

where $\boldsymbol{q} \in \mathcal{C}$ is the configuration vector, $\mathcal{C} \subset \mathbb{R}^{n}$ is the configuration space, $\boldsymbol{u} \in \mathcal{U}$ is the vector of $m<n$ control inputs, $\mathcal{U} \subset \mathbb{R}^{m}$ is the control space and $f: \mathbb{R}^{n} \times \mathbb{R}^{m} \rightarrow \mathbb{R}^{n}$ is a vector-valued map describing the system dynamics. The configuration vector $\boldsymbol{q}=\left[\begin{array}{lll}q_{1} & \cdots & q_{n}\end{array}\right]^{T}$ is the vector of generalized coordinates. Assume that (1) is subject to $\kappa<n$ constraint equations. Each constraint $i$ is of the form

$$
\sum_{j=1}^{n} a_{i j}\left(q_{1}, \ldots, q_{n}, t\right) \dot{q}_{j}+b_{i}\left(q_{1}, \ldots, q_{n}, t\right)=0 .
$$

The differential or Pfaffian form of (2) is

$$
\sum_{j=1}^{n} a_{i j}\left(q_{1}, \ldots, q_{n}, t\right) \mathrm{d} q_{j}+b_{i}\left(q_{1}, \ldots, q_{n}, t\right) \mathrm{d} t=0,
$$

and represents a restriction on the amount by which the generalized coordinates may change in a time interval $\mathrm{d} t$. We consider the class of nonlinear systems described by (1), which are subject to $\kappa$ nonholonomic, time-independent constraints (2). The constraint equations are written in compact form as

$$
\boldsymbol{A}(\boldsymbol{q}) \dot{\boldsymbol{q}}=\boldsymbol{b}(\boldsymbol{q}),
$$

and may refer to either kinematic or dynamic nonholonomic systems. Due to space limitations, this paper describes the construction of dipole-like fields and their connection to systems with catastatic Pfaffian constraints $(\boldsymbol{b}(\boldsymbol{q})=\mathbf{0})$ [36].

\section{The Dipole-Like Vector Field}

The physical electric dipole is a pair of point charges of equal magnitude but opposite sign; $Q_{1}=+Q, Q_{2}=-Q$, separated by a distance $2 a, a>0$. Let us consider an electric dipole in a workspace $\mathcal{W} \subseteq \mathbb{R}^{2}$, with the charges located at

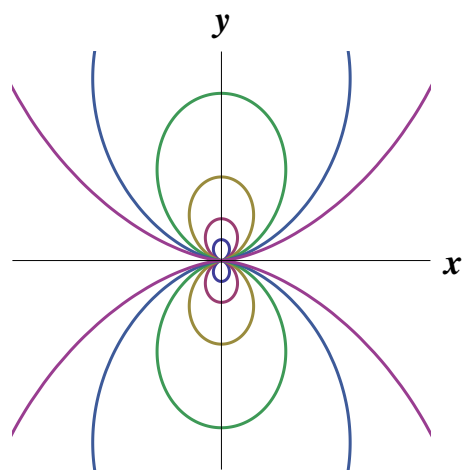

Fig. 1. Flow lines of the Electric Point Dipole.

$\boldsymbol{r}_{Q_{1}}=\left[\begin{array}{ll}a & 0\end{array}\right]^{T}$ and $\boldsymbol{r}_{Q_{2}}=\left[\begin{array}{ll}-a & 0\end{array}\right]^{T}$ respectively, where $\boldsymbol{r}_{Q_{i}} \in \mathbb{R}^{2}$ is the position vector of the charge $i$ with respect to the origin. The dipole moment $\boldsymbol{p}$ of the physical dipole is defined as $\boldsymbol{p}=Q_{1} \boldsymbol{r}_{Q_{1}}+Q_{2} \boldsymbol{r}_{Q_{2}}=Q\left(\boldsymbol{r}_{Q_{1}}-\boldsymbol{r}_{Q_{2}}\right)=Q \boldsymbol{d}$, where $\boldsymbol{d}$ is the vector from the negative to the positive charge.

The point dipole is an idealization of the physical dipole, obtained as the distance between the charges tends to zero, $\boldsymbol{d} \rightarrow \mathbf{0}$, while the dipole moment $\boldsymbol{p}$ is kept constant. Using polar coordinates, the intensity of this electric field at a point $A$ is written as [37]

$$
\boldsymbol{E}_{\mathrm{dip}}(r, \theta)=\left(\frac{2 p \cos \theta}{4 \pi \varepsilon_{0} r^{3}}\right) \hat{\boldsymbol{r}}+\left(\frac{p \sin \theta}{4 \pi \varepsilon_{0} r^{3}}\right) \hat{\boldsymbol{\theta}},
$$

where $(r, \theta)$ are the polar coordinates of the position vector $r$ of point $A$ and $\varepsilon_{0}$ is a constant. From the definition of a flow line one has

$$
\frac{\mathrm{d} r}{r \mathrm{~d} \theta}=\frac{E_{r}}{E_{\theta}} \Rightarrow \frac{\mathrm{d} r}{r \mathrm{~d} \theta}=\frac{2 \cos \theta}{\sin \theta} \Rightarrow \frac{1}{r} \mathrm{~d} r=\frac{2 \cos \theta}{\sin \theta} \mathrm{d} \theta,
$$

which yields $r=R \sin ^{2} \theta$, where $R>0$ is a constant associated with the particular flow line [38]. Thus, as the distance to the origin tends to zero, $r \rightarrow 0$, then $\sin \theta \rightarrow 0 \Rightarrow$ $\theta \rightarrow 0$ if $x \geq 0$, or $\theta \rightarrow \pi$ if $x<0$, i.e. the flow lines of the point dipole converge to the origin with direction parallel to $\mathrm{x}$-axis, see Fig. 1. The flow lines of a point dipole having a moment $\boldsymbol{p}$ of polar coordinates $\left(p, \theta_{1}\right)$ always converge to the origin with direction parallel to $\boldsymbol{p}$, since they are described by $r=R \sin ^{2}\left(\theta-\theta_{1}\right)$. In general, the field of the electric point dipole, defined by a dipole moment $\boldsymbol{p} \in \mathbb{R}^{3}$ in a workspace $\mathcal{W} \subset \mathbb{R}^{3}$ is [37]

$$
\boldsymbol{E}_{\mathrm{dip}}(\boldsymbol{r})=\frac{1}{4 \pi \varepsilon_{0} r^{3}}(3(\boldsymbol{p} \cdot \hat{\boldsymbol{r}}) \hat{\boldsymbol{r}}-\boldsymbol{p})-\frac{1}{3 \varepsilon_{0}} \boldsymbol{p} \delta^{3}(\boldsymbol{r}),
$$

where $\delta^{3}(\cdot)$ is a 3-dimensional Dirac function. Using spherical coordinates, one can show that if the field is axialsymmetric, then the flow lines converge to the origin, parallel with the dipole moment $\boldsymbol{p}$.

The behavior of the dipole flow lines around the origin is the motivation for the control strategy adopted in this paper. Assume that the dipole moment $\boldsymbol{p} \in \mathbb{R}^{2}$ is assigned at a goal position $\boldsymbol{r}_{G}=\left[\begin{array}{ll}x_{G} & y_{G}\end{array}\right]^{T}$ such that its direction coincides with a desired orientation $\theta_{G}$. Then, the resulting flow lines converge to the goal position $\boldsymbol{r}_{G}$ with direction 
$\theta_{G} \pm \xi \pi, \xi=0,1$. Then, one could claim that the control objective for the system (1), where $\boldsymbol{q}=\left[\begin{array}{lll}x & y & \theta\end{array}\right]^{T}$, is to design a feedback control law $\boldsymbol{u}=\gamma(\boldsymbol{q})$ such that the system reaches the desired configuration $\boldsymbol{q}_{\boldsymbol{G}}=\left[\begin{array}{lll}x_{G} & y_{G} & \theta_{G}\end{array}\right]^{T}$ by following the flow lines as reference paths.

Following (7), the dipole-like vector field is proposed

$$
\mathbf{F}(\boldsymbol{q})=\overbrace{\lambda(\boldsymbol{p} \cdot \boldsymbol{q}) \boldsymbol{q}-\boldsymbol{p}}^{\mathbf{F}_{1}(\boldsymbol{q})}+\overbrace{\boldsymbol{p} \exp ^{-\|q\|^{2}}}^{\mathbf{F}_{2}(\boldsymbol{q})}
$$

where $\boldsymbol{q} \in \mathbb{R}^{n}$ is the vector of generalized coordinates or the system state vector, $\boldsymbol{p} \in \mathbb{R}^{n}$ is the dipole moment, $\|q\|$ is the Euclidean norm of $\boldsymbol{q}$ and $\lambda>0$ is a constant. The vector field $\mathbf{F}_{\mathbf{1}}(\boldsymbol{q})$ defines the direction of the flow lines to be consistent with the point dipole flow lines, whereas the vector field $\mathbf{F}_{\mathbf{2}}(\boldsymbol{q})$ expresses the $n$-dimensional Dirac function.

\section{Dipole-Like Vector FiELdS AND NonholonomiC SYSTEMS}

\section{A. The Unicycle}

Consider the motion of a robot in a bounded workspace $\mathcal{W} \subset \mathbb{R}^{2}$, which is described by the unicycle kinematics

$$
\left[\begin{array}{c}
\dot{x} \\
\dot{y} \\
\dot{\theta}
\end{array}\right]=\left[\begin{array}{c}
\cos \theta \\
\sin \theta \\
0
\end{array}\right] u_{1}+\left[\begin{array}{l}
0 \\
0 \\
1
\end{array}\right] u_{2}
$$

where $\boldsymbol{q}=\left[\begin{array}{lll}x & y & \theta\end{array}\right]^{T} \in \mathcal{C} \subset \mathbb{R}^{3}$ is the configuration vector, $\mathcal{C}$ is a smooth manifold denoting the configuration space of the robot, $x, y$ are the position coordinates and $\theta$ is the orientation of the robot with respect to a cartesian frame $\mathcal{G}$. The control inputs are $u_{1}$ and $u_{2}$, i.e. the linear and the angular velocity of the robot, respectively. Denote $\boldsymbol{u}=$ $\left[\begin{array}{ll}u_{1} & u_{2}\end{array}\right]^{T} \in \mathcal{U} \subset \mathbb{R}^{2}$ and $\mathbf{g}_{\mathbf{1}}(\boldsymbol{q})=\left[\begin{array}{ccc}\cos \theta & \sin \theta & 0\end{array}\right]^{T}$, $\mathbf{g}_{2}=\left[\begin{array}{lll}0 & 0 & 1\end{array}\right]^{T}$. System (9) is subject to $\kappa=1$ nonholonomic catastatic constraint

$$
\underbrace{[\sin \theta-\cos \theta 0]}_{\boldsymbol{a}^{T}(\boldsymbol{q})}\left[\begin{array}{c}
\dot{x} \\
\dot{y} \\
\dot{\theta}
\end{array}\right]=0 \Rightarrow \boldsymbol{a}^{T}(\boldsymbol{q}) \dot{\boldsymbol{q}}=0, \boldsymbol{a}^{T}(\boldsymbol{q}) \mathrm{d} \boldsymbol{q}=0 .
$$

The constraint implies that the subspace of the infinitesimal displacements $\mathrm{d} \boldsymbol{q}$ at fixed $\boldsymbol{q}$ is given as the kernel (or null space) of $\boldsymbol{a}^{T}(\boldsymbol{q})$.

Let us now assume that the goal configuration $\boldsymbol{q}_{\boldsymbol{G}}$ is the origin. The constraint equation at $\boldsymbol{q}_{\boldsymbol{G}}$ reads

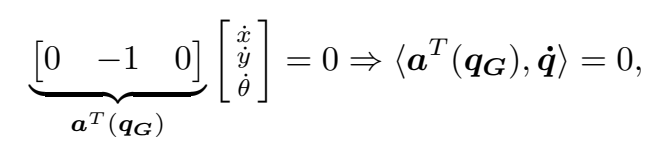

where $\langle\cdot, \cdot\rangle$ stands for the inner product. Thus, the vector of generalized velocities $\dot{\boldsymbol{q}}$ at the goal configuration $\boldsymbol{q}_{\boldsymbol{G}}$ is restricted to lie in the $n-\kappa=2$ dimensional subspace (hyperplane) $\mathcal{V}$ of the tangent space $T_{\boldsymbol{q}_{\boldsymbol{G}}}(\mathcal{C})$ of $\mathcal{C}$ at $\boldsymbol{q}_{\boldsymbol{G}}$. The hyperplane $\mathcal{V}$ is the set of all $\dot{\boldsymbol{q}}=\left[\begin{array}{lll}\dot{x} & 0 & \dot{\theta}\end{array}\right]^{T}$ satisfying the constraint at the goal configuration $\boldsymbol{q}_{\boldsymbol{G}}$, see Fig. 2. Thus, only the $n-\kappa$ components of $\dot{\boldsymbol{q}}$ can be chosen independently, whereas the remaining $\kappa$ need to be set to satisfy the Pfaffian constraints [39].

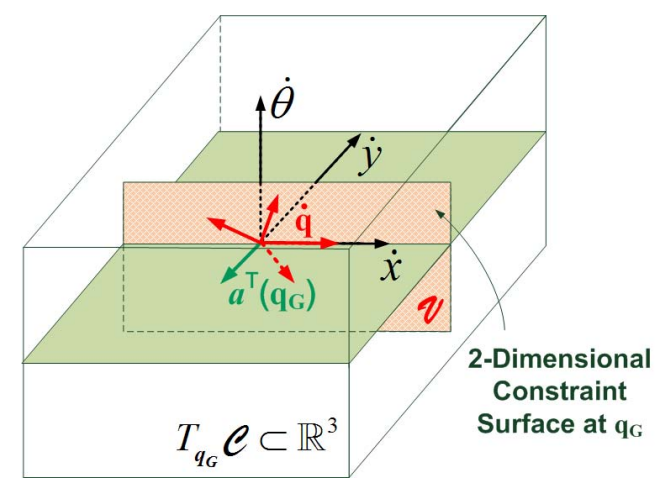

Fig. 2. The hyperplane $\mathcal{V}$ serves locally as the $n-\kappa$ dimensional constraint surface at the goal configuration $\boldsymbol{q}_{\boldsymbol{G}}$, whose normal is the constraint vector $\boldsymbol{a}^{T}\left(\boldsymbol{q}_{\boldsymbol{G}}\right)$. The allowed generalized velocities $\dot{\boldsymbol{q}}$ at $\boldsymbol{q}_{\boldsymbol{G}}$ lie tangent to $\mathcal{V}$.

Having a geometric interpretation of the Pfaffian constraint, one is interested in designing a dipole-like field $\mathbf{F}(\boldsymbol{q})$ such that its flow lines

- converge to the goal configuration $q_{G}$,

- are consistent with the constrained dynamics.

We call a flow line consistent if its tangent vector satisfies the Pfaffian constraint. For the unicycle, the flow lines of a 3-dimensional vector field $\mathbf{F}(x, y, \theta)=\mathrm{F}_{x} \hat{\boldsymbol{x}}+\mathrm{F}_{y} \hat{\boldsymbol{y}}+\mathrm{F}_{\theta} \hat{\boldsymbol{\theta}}$ should satisfy

$$
\underbrace{[\sin \theta-\cos \theta 0}_{\boldsymbol{a}^{T}(\boldsymbol{q})}]\left[\begin{array}{l}
\mathrm{F}_{x} \\
\mathrm{~F}_{y} \\
\mathrm{~F}_{\theta}
\end{array}\right]=0 \Rightarrow \mathrm{F}_{x} \sin \theta-\mathrm{F}_{y} \cos \theta=0 .
$$

Note that the $\mathrm{F}_{\theta}$ component of the field does not affect whether the constraint is satisfied. In this case, one can define a $N=2$ dimensional dipole-like field, in terms of $\mathrm{F}_{x}, \mathrm{~F}_{y}$ only, with $\mathrm{F}_{\theta}=0$. (This decision is system-dependent.)

The field is generated by a dipole moment $\boldsymbol{p} \in \mathbb{R}^{N}$ so that the flow lines converge to $\boldsymbol{q}_{\boldsymbol{G}}$ having tangent vectors that satisfy the Pfaffian constraint. This condition is equivalent with the requirement that the vector $\boldsymbol{p}$ satisfies the Pfaffian constraint at $\boldsymbol{q}_{\boldsymbol{G}},\left\langle\boldsymbol{a}^{T}\left(\boldsymbol{q}_{\boldsymbol{G}}\right), \boldsymbol{p}\right\rangle=0$, which holds for $\boldsymbol{p}=\left[\begin{array}{lll}p & 0 & \theta\end{array}\right]^{T}$, for any $\theta$. Nevertheless, since the field is defined in a $N=2$ dimensional subspace of $\mathbb{R}^{n}$, one can simply choose $\boldsymbol{p}=\left[\begin{array}{ll}p & 0\end{array}\right]^{T} \in \mathbb{R}^{N}$, neglecting thus the generalized coordinate that is not involved with the satisfiability of the nonholonomic constraint. Taking (7), and ignoring the $\frac{p}{4 \pi \varepsilon_{0} r^{3}}$ factor, one gets a simple choice of a dipole-like vector field:

$$
\mathrm{F}_{x}=2 \cos ^{2} \phi-\sin ^{2} \phi, \quad \mathrm{F}_{y}=3 \sin \phi \cos \phi, \quad \mathrm{F}_{\theta}=0,
$$

with $(r, \phi)$ being the polar representation of $(x, y)$. The flow line equation is $\frac{d r}{d \phi}=\frac{2 r \cos \phi}{\sin \phi}$, and assuming that the unicycle vector field is initially aligned to the flow line tangent vector, implying $3 x y \cos \theta+\left(y^{2}-2 x^{2}\right) \sin \theta=0$, we can verify that the flow line is consistent, by computing the derivative of the above condition and verifying that the choice of inputs

$$
\begin{aligned}
& u_{1}=-y\left[\left(y^{2}-2 x^{2}\right) \cos \theta-3 x y \sin \theta-4 x \sin \theta \cos \theta\right], \\
& u_{2}=2 x^{2} \sin ^{2} \theta+y^{2} \cos ^{2} \theta+1 \neq 0,
\end{aligned}
$$


keeps the system on the flow line. To design the stabilizing control law, define the projection of $\mathbf{F}(\boldsymbol{q})$ on $\boldsymbol{a}(\boldsymbol{q})$ as the system output,

$$
h(\boldsymbol{q}) \triangleq\left\langle\boldsymbol{a}^{T}, \mathbf{F}\right\rangle=0.5[3 \sin (2 \phi-\theta)-\sin \theta],
$$

and seek a control law that stabilizes $h(\boldsymbol{q})$ to zero. One can verify that the choice of $u_{2}$

$$
\begin{aligned}
u_{2}= & \frac{2 k h(\boldsymbol{q})}{3 \cos (2 \phi-\theta)+\cos \theta} \\
& -\frac{6 u_{1}(x \sin \theta-y \cos \theta) \cos (2 \phi-\theta)}{r^{2}(3 \cos (2 \phi-\theta)+\cos \theta)},
\end{aligned}
$$

with $k>0$, renders the closed loop dynamics of $h(\boldsymbol{q})$ exponentially stable: $\dot{h}=-k h$. Note that in (12), the term $3 \cos (2 \phi-\theta)+\cos \theta$ appearing in the denominator may become zero. To avoid this singularity one may resort to switching and set:

$u_{2}= \begin{cases}\frac{2 k h(\boldsymbol{q})-6 u_{1} r^{-2}(x \sin \theta-y \cos \theta) \cos (2 \phi-\theta)}{r^{2}(3 \cos (2 \phi-\theta)+\cos \theta)}, & |3 \cos (2 \phi-\theta)+\cos \theta|>\epsilon \\ k\left(\theta_{d}-\theta\right), & |3 \cos (2 \phi-\theta)+\cos \theta| \leq \epsilon,\end{cases}$

where $\theta_{d}$ denotes the direction of $\mathbf{F}$, and $\epsilon$ is a small constant. Setting arbitrarily a constantly positive forward speed for the unicycle, $u_{1}=\tanh r,{ }^{1}$ one obtains a closed loop system that converges to $\boldsymbol{q}_{\boldsymbol{G}}$ following the flow lines of $\mathbf{F}$ (see Fig. 3, $4,5)$.

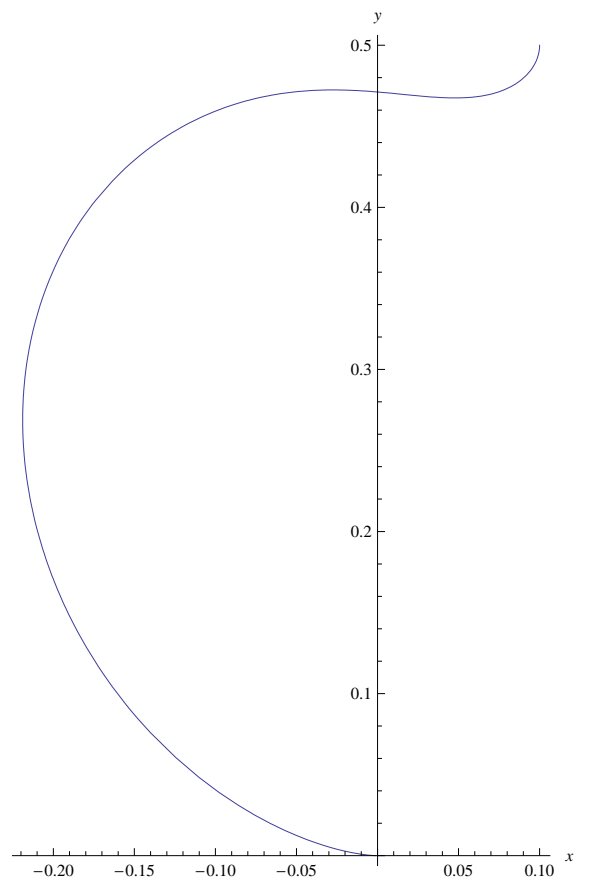

Fig. 3. Closed loop behavior of a unicycle, initialized at $(x, y, \theta)=$ $\left(0.1,0.5,-\frac{\pi}{2}\right)$, with a control law forcing it to follow the flow lines of a dipole-like vector field.

\footnotetext{
${ }^{1}$ Although it is possible to set $u_{1}$ as proportional to $r^{2}$, to cancel the effect of $r^{2}$ in the denominator of (12), the choice made here yields faster convergence.
}

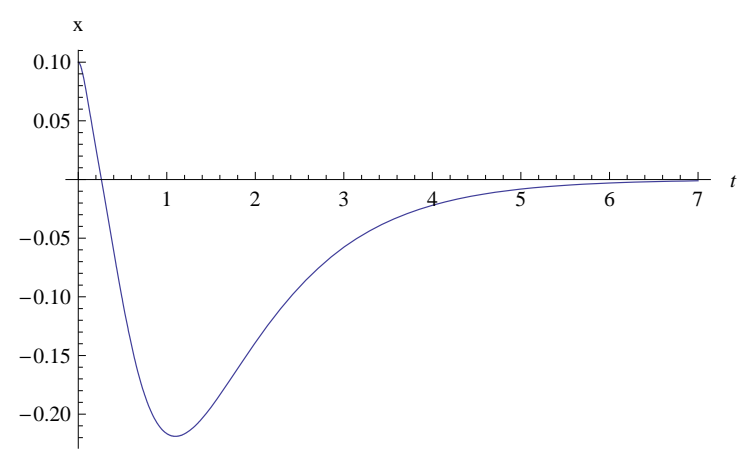

(a) Trajectory for $x$.

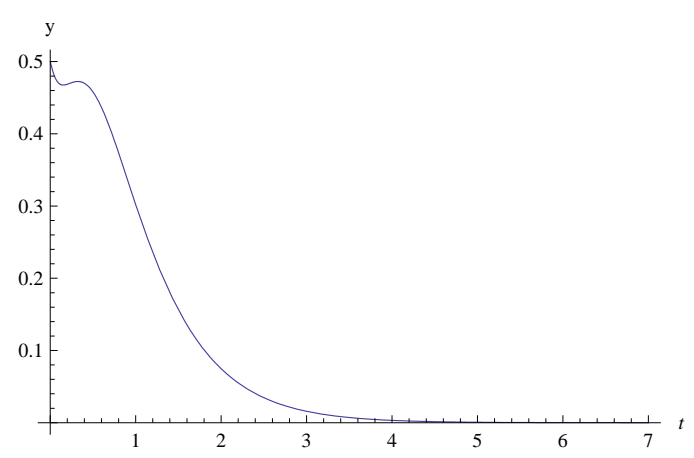

(b) Trajectory for $y$.

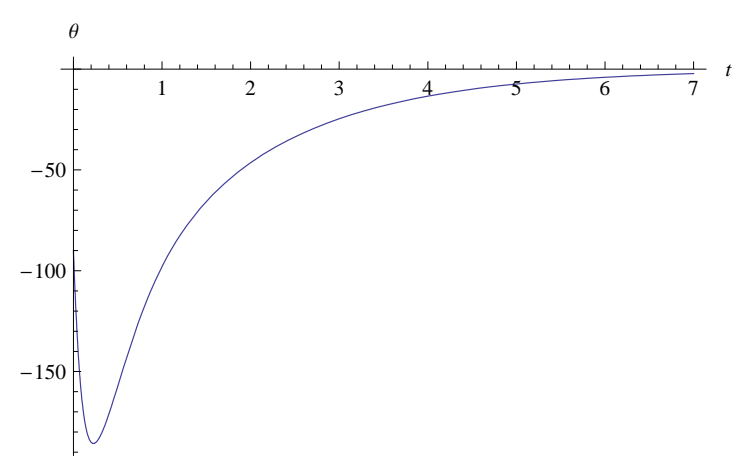

(c) Trajectory for $\theta$.

Fig. 4. State trajectories for the unicycle tracing the path shown in Fig. 3.

\section{B. Brockett's Nonholonomic Double Integrator}

Consider the nonholonomic double integrator

$$
\left[\begin{array}{c}
\dot{x}_{1} \\
\dot{x}_{2} \\
\dot{x}_{3}
\end{array}\right]=\left[\begin{array}{c}
1 \\
0 \\
-x_{2}
\end{array}\right] u_{1}+\left[\begin{array}{c}
0 \\
1 \\
x_{1}
\end{array}\right] u_{2}
$$

where $\boldsymbol{q}=\left[\begin{array}{lll}x_{1} & x_{2} & x_{3}\end{array}\right]^{T}$ is the state vector, $\mathbf{g}_{\mathbf{1}}(\boldsymbol{q})=$ $\left[\begin{array}{lll}1 & 0 & -x_{2}\end{array}\right]^{T}$ and $\mathbf{g}_{2}(\boldsymbol{q})=\left[\begin{array}{lll}0 & 1 & x_{1}\end{array}\right]^{T}$ are the control vector fields. The $\kappa=1$ nonholonomic constraint is

$$
\underbrace{\left[\begin{array}{lll}
x_{2} & -x_{1} & 1
\end{array}\right]}_{\boldsymbol{a}^{T}(\boldsymbol{q})}\left[\begin{array}{c}
\dot{x}_{1} \\
\dot{x}_{2} \\
\dot{x}_{3}
\end{array}\right]=0 \Rightarrow\left\langle\boldsymbol{a}^{T}(\boldsymbol{q}), \dot{\boldsymbol{q}}\right\rangle=0 .
$$

The constraint vector at the origin is $\boldsymbol{a}^{T}\left(\boldsymbol{q}_{\boldsymbol{G}}\right)=\left[\begin{array}{lll}0 & 0 & 1\end{array}\right]$. Thus, generalized velocities $\dot{\boldsymbol{q}}$ are restricted to lie in the $n-$ $\kappa=2$ dimensional hyperplane $\mathcal{V}$ of the tangent space $T_{\boldsymbol{q}_{\boldsymbol{G}}}(\mathcal{C})$ 


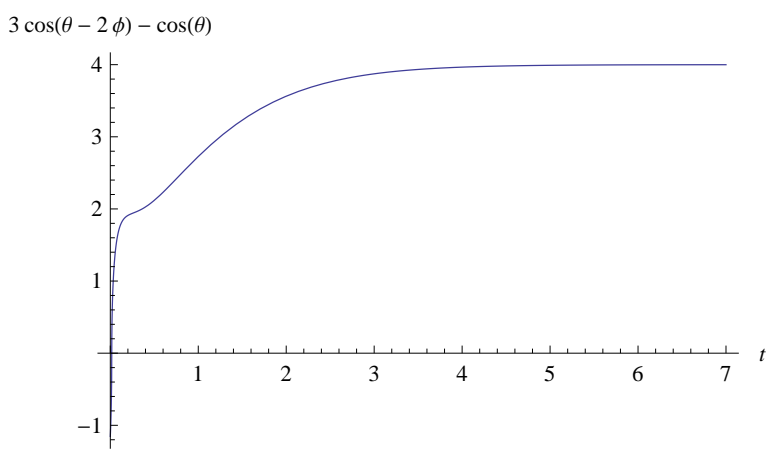

(a) The denominator in (12).

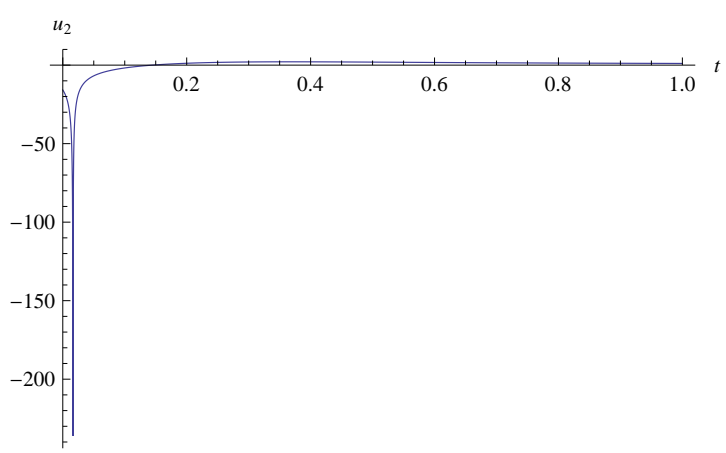

(b) Control input $u_{2}$.

Fig. 5. As the denominator expression in (12) crosses zero from negative to positive values, near simulation time 0.04 , the control input $u_{2}$ switches between the two branches of (5). What keeps the input $u_{2}$ bounded is that it is given by the lower branch of (5) for that short time period of the transition. Here $\epsilon$ is set at $10^{-3}$.

at $\boldsymbol{q}_{\boldsymbol{G}}$, which is the set of all $\dot{\boldsymbol{q}}=\left[\begin{array}{lll}\dot{x}_{1} & \dot{x}_{2} & 0\end{array}\right]^{T}$ that satisfies (15), see Fig. 6. The field $\mathbf{F}(\boldsymbol{q})=\mathrm{F}_{x_{1}} \hat{\boldsymbol{x}}_{\mathbf{1}}+\mathrm{F}_{x_{2}} \hat{\boldsymbol{x}}_{\mathbf{2}}+\mathrm{F}_{x_{3}} \hat{\boldsymbol{x}}_{\mathbf{3}}$

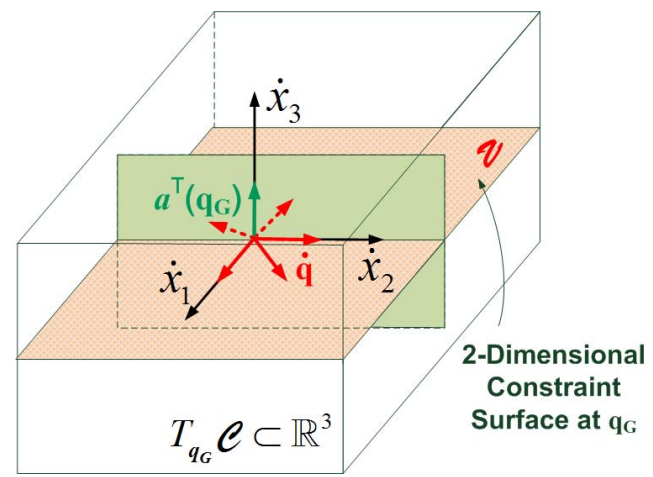

Fig. 6. The hyperplane $\mathcal{V}$ serves locally as the $n-\kappa$ dimensional constraint surface at the goal configuration $\boldsymbol{q}_{\boldsymbol{G}}$, whose normal is the constraint vector $\boldsymbol{a}^{T}\left(\boldsymbol{q}_{\boldsymbol{G}}\right)$. The allowed generalized velocities $\dot{\boldsymbol{q}}$ at $\boldsymbol{q}_{\boldsymbol{G}}$ lie tangent to $\mathcal{V}$.

should satisfy the constraint equation

$$
\underbrace{\left[x_{2}-x_{1} 1\right.}_{\boldsymbol{a}^{T}(\boldsymbol{q})}]\left[\begin{array}{c}
\mathrm{F}_{x_{1}} \\
\mathrm{~F}_{x_{2}} \\
\mathrm{~F}_{x_{3}}
\end{array}\right]=0 \Rightarrow \mathrm{F}_{x_{1}} x_{2}-\mathrm{F}_{x_{2}} x_{1}+\mathrm{F}_{x_{3}}=0 .
$$

In this case, (16) requires a $N=3$ dimensional dipolelike field, in terms of $\mathrm{F}_{x_{1}}, \mathrm{~F}_{x_{2}}, \mathrm{~F}_{x_{3}}$, that is dependent on the full state vector $\boldsymbol{q}$. A dipole moment $\boldsymbol{p}$ satisfying the nonholonomic constraint at the goal configuration $\boldsymbol{q}_{\boldsymbol{G}}$ gives
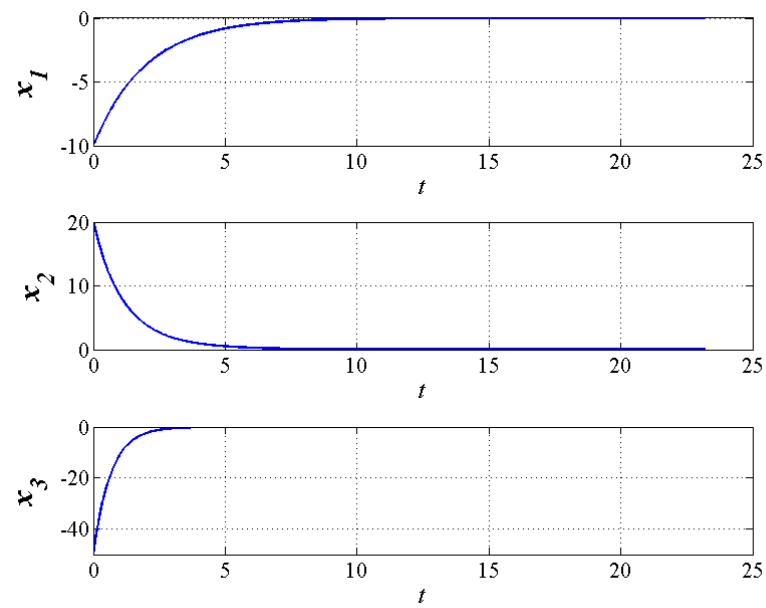

Fig. 7. Response of $x_{1}, x_{2}, x_{3}$ under the control law (17). $x_{3}$ converges to zero faster than $x_{1}, x_{2}$, as expected. Actually, $x_{3} \rightarrow 0$ exponentially, as verified by the closed loop dynamics: $\dot{x}_{3}=\left(-k+k_{1}\right) x_{3}$, where $k_{1}<k$.

$\left\langle\boldsymbol{a}^{T}\left(\boldsymbol{q}_{\boldsymbol{G}}\right), \boldsymbol{p}\right\rangle=0$, which holds for $\boldsymbol{p}=\left[\begin{array}{lll}p_{1} & p_{2} & 0\end{array}\right]^{T}, p_{1}$, $p_{2} \in \mathbb{R}$. With $\boldsymbol{p}=\left[\begin{array}{lll}1 & 0 & 0\end{array}\right]^{T}$ and $\lambda=1$, (8) yields: $\mathrm{F}_{x_{1}}=$ $x_{1}^{2}-1+\exp ^{-\left(x_{1}^{2}+x_{2}^{2}+x_{3}^{2}\right)}, \mathrm{F}_{x_{2}}=x_{1} x_{2}, \mathrm{~F}_{x_{3}}=x_{1} x_{3}$. The field is symmetric with respect to the axis of the dipole moment $\boldsymbol{p}$. Condition (16) can serve as an output to be regulated, $h(\boldsymbol{q}) \rightarrow 0$, so that the system vector field is aligned with the dipole field. By substituting the field components $\mathrm{F}_{x_{1}}, \mathrm{~F}_{x_{2}}$, $\mathrm{F}_{x_{3}}$ in (16) one has

$$
h(\boldsymbol{q})=x_{1} x_{3}-x_{2}\left[1-\exp ^{-\left(x_{1}^{2}+x_{2}^{2}+x_{3}^{2}\right)}\right] .
$$

This condition suggests that the system can be stabilized with a strategy that brings first $x_{3} \rightarrow 0$ and then $x_{2}, x_{1} \rightarrow 0$, i.e. in a way such that $x_{3}$ converges faster than $x_{1}, x_{2}$ to zero. This is consistent with other relevant control designs for Brockett's integrator [19], [20].

A simplified field of the form $\mathbf{F}(\boldsymbol{q})=\lambda(\boldsymbol{p} \cdot \boldsymbol{q}) \boldsymbol{q}$, where $\lambda=1, \mathrm{~F}_{x_{1}}=x_{1}^{2}, \mathrm{~F}_{x_{2}}=x_{1} x_{2}, \mathrm{~F}_{x_{3}}=x_{1} x_{3}$, enables an easier controller design, since now $h(\boldsymbol{q})=x_{1} x_{3}$. Then, if $x_{1} \neq 0$, choosing $u_{2}=-k \frac{x_{3}}{x_{1}}-\frac{x_{3}-x_{2} x_{1}}{x_{1}^{2}} u_{1}$, where $k>0$ and $u_{1}=-k_{1} x_{1}$, where $k_{1}>0$, results in $\dot{h}=-k h$. Since $x_{1}=0$ results in $\mathbf{F}=\mathbf{0}$, a stabilizing switching strategy can be formulated as:

$$
\begin{aligned}
& u_{1}= \begin{cases}-k_{1} x_{1}, & \text { if } x_{1} \neq 0 \\
-k_{3} x_{3}, & \text { if } x_{1}=0\end{cases} \\
& u_{2}=\left\{\begin{array}{cc}
-k \frac{x_{3}}{x_{1}}-\frac{x_{3}-x_{2} x_{1}}{x_{1}^{2}} u_{1}, & \text { if } x_{1} \neq 0 \\
-k_{2} x_{2}, & \text { if } x_{1}=0
\end{array}\right.
\end{aligned}
$$

where $k_{2}, k_{3}>0$. The closed loop system response is given in Fig. 7.

\section{Conclusions}

The goal of this paper is to suggest a framework for motion planning of systems with Pfaffian constraints, in which guidelines for a stabilizing control design can be established in a uniform way across different dynamics. The control law 
examples presented, therefore, may not claim performance but rather demonstrate that the same design rules can be used for different systems. The basic idea is that one can define a dipole-like vector field, the flow lines of which are consistent with the constraints imposed, and then regulate to zero an output expressing the misalignment between the system vector field and the dipole-like field, in order to stabilize the constrained dynamics. Preliminary work suggests that this idea may be compatible with artificial potential field methods used for robot navigation, in which case the development of a new, unified framework for stabilization under combined holonomic and nonholonomic constraints may be feasible.

\section{REFERENCES}

[1] R. W. Brockett, "Asymptotic stability and feedback stabilization," in Differential Geometric Control Theory. R.W. Brockett, R.S. Millman and H.J. Sussman, Eds., Boston, Birkhauser, 1983, pp. 181-191.

[2] A. M. Bloch, M. Reyhanoglu, and N. H. McClamroch, "Control and stabilization of nonholonomic dynamic systems," IEEE Transactions on Automatic Control, vol. 37, no. 11, pp. 1746-1757, Nov. 1992.

[3] C. Canudas de Wit and O. J. Sordalen, "Exponential stabilization of mobile robots with nonholonomic constraints," IEEE Transactions on Automatic Control, vol. 37, no. 11, pp. 1791-1797, Nov. 1992.

[4] J.-B. Pomet, "Explicit design of time-varying stabilizing control laws for a class of controllable systems without drift," Systems and Control Letters, vol. 18, pp. 147-158, 1992.

[5] O. J. Sordalen and O. Egeland, "Exponential stabilization of nonholonomic chained systems," IEEE Transactions on Automatic Control, vol. 40, no. 1, pp. 35-48, Jan. 1995.

[6] R. M'Closkey and R. Murray, "Exponential stabilization of driftless nonlinear control systems using homogeneous feedback," IEEE Transactions on Automatic Control, vol. 42, no. 5, pp. 614-628, 1997.

[7] J.-M. Godhavn and O. Egeland, "A lyapunov approach to exponential stabilization of nonholonomic systems in power form," IEEE Transactions on Automatic Control, vol. 42, no. 7, pp. 1028-1032, Jul. 1997.

[8] Y.-P. Tian and S. Li, "Exponential stabilization of nonholonomic dynamic systems by smooth time-varying control," Automatica, vol. 38, pp. 1138-1143, 2002.

[9] M. Reyhanoglu, "On the stabilization of a class of nonholonomic systems using invariant manifold technique," in Proc. of the 34th IEEE Conference on Decision and Control, New Orleans, LA, USA, Dec. 1995, pp. 2125-2126.

[10] A. Astolfi, "Discontinuous control of nonholonomic systems," Systems and Control Letters, vol. 27, no. 1, pp. 37-45, Jan. 1996.

[11] A. Bloch, S. Drakunov, and M. Kinyon, "Nonholonomic stabilization and isospectral flows," in Proc. of the 37th IEEE Conference on Decision and Control, Tampa, FL, USA, Dec. 1998, pp. 3581-3586.

[12] I. Kolmanovsky, M. Reyhanoglu, and N. H. McClamroch, "Switched mode feedback control laws for nonholonomic systems in extended power form," Systems and Control Letters, vol. 27, no. 1, pp. 29-36, Jan. 1996.

[13] J. P. Hespanha and A. S. Morse, "Stabilization of non-holonomic integrators via logic-based switching," Automatica, vol. 35, no. 3, pp. 385-393, Mar. 1999.

[14] M. Reyhanoglu, A. van der Schaft, N. H. McClamroch, and I. Kolmanovsky, "Dynamics and control of a class of underactuated mechanical systems," IEEE Transactions on Automatic Control, vol. 44, no. 9, pp. 1663-1671, Sep. 1999.

[15] M. Chun-Yi Su, Y. Stepanenko, and A. A. Goldenberg, "Reduced order model and robust control architecture for mechanical systems with nonholonomic pfaffian constraints," IEEE Transactions on Systems, Man, and Cybernetics - Part A: Systems and Human, vol. 29, no. 3, pp. 307-313, May 1999.

[16] R. Colbaugh, E. Barany, and K. Glass, "Adaptive stabilization of nonholonomic mechanical systems," in Proc. of the 36th IEEE Conference on Decision and Control, San Diego, CA, USA, Dec. 1997, pp. 30813088.

[17] Z.-P. Jiang, "A unified lyapunov framework for stabilization and tracking of nonholonomic systems," in Proc. of the 38th IEEE Conference on Decision and Control, Phoenix, Arizona, USA, Dec. 1999, pp. 2088-2093.
[18] W. Dong, Y. Xu, and W. Huo, "On stabilization of uncertain dynamic nonholonomic systems," International Journal of Control, vol. 73, no. 4 , pp. $349-359,2000$.

[19] A. Bloch and S. Drakunov, "Stabilization and tracking in the nonholonomic integrator via sliding modes," Systems and Control Letters, vol. 29, no. 1, pp. 91-99, 1996.

[20] A. Astolfi, "Discontinuous control of the Brockett integrator," in Proc. of the 36th IEEE Conference on Decision and Control, San Diego, CA, USA, Dec. 1997, pp. 4334-4339.

[21] A. Bloch, S. Drakunov, and M. Kinyon, "Stabilization of brockett's generalized canonical driftless system," in Proc. of the 36th IEEE Conference on Decision and Control, San Diego, CA, USA, Dec. 1997, pp. $4260-4265$.

[22] N. Khaneja and R. Brockett, "Dynamic feedback stabilization of nonholonomic systems," in Proc. of the 38th IEEE Conference on Decision and Control, Phoenix, Arizona, USA, Dec. 1999, pp. 1640-1645.

[23] H. Choset, K. Lynch, S. Hutchinson, G. Kantor, W. Burgard, L. Kavraki, and S. Thrun, Principles of Robot Motion. MIT Press, 2005.

[24] O. Khatib, "Real-time obstacle avoidance for manipulators and mobile robots," International Journal of Robotic Research, vol. 5, no. 1, pp. 90-98, Spring, 1986.

[25] E. Rimon and D. Koditschek, "Exact robot navigation using artificial potential functions," IEEE Transactions on Robotics and Automation, vol. 8, no. 5, pp. 501-518, Oct. 1992.

[26] S. Sekhavat and M. Chyba, "Nonholonomic deformation of a potential field for motion planning," in IEEE International Conference on Robotics and Automation, 1999, pp. 817-822.

[27] H. G. Tanner and K. J. Kyriakopoulos, "Nonholonomic motion planning for mobile manipulators," in Proc. of the 2000 IEEE International Conference on Robotics and Automation, San Francisco, CA, Apr. 2000, pp. 1233-1238.

[28] D. V. Dimarogonas and K. J. Kyriakopoulos, "A feedback control scheme for multiple independent dynamic non-point agents," International Journal of Control, vol. 79, no. 12, pp. 1613-1623, Dec. 2006.

[29] S. G. Loizou and K. J. Kyriakopoulos, "Navigation of multiple kinematically constrained robots," IEEE Transactions on Robotics, vol. 24, no. 1, pp. 221-231, Feb. 2008.

[30] G. P. Roussos, D. V. Dimarogonas, and K. J. Kyriakopoulos, "Distributed 3D navigation and collision avoidance for nonholonomic aircraft-like vehicles," in Proc. of the European Control Conference 2009, Budapest, Hungary, Aug. 2009, pp. 1830-1835.

[31] G. A. D. Lopes and D. E. Koditschek, "Visual servoing for nonholonomically constrained three degree of freedom kinematic systems," The International Journal of Robotics Research, vol. 26, no. 7, Jul. 2007.

[32] A. A. Masoud, "A harmonic potential field approach for navigating a rigid, nonholonomic robot in a cluttered environment," in Proc. of the 2009 IEEE International Conference on Robotics and Automation, Kobe, Japan, May 2009, pp. 3993-3999.

[33] S. R. Lindemann and S. M. LaValle, "Smoothly blending vector fields for global robot navigation," in Proc. of the 44th IEEE Conference on Decision and Control and the 2005 European Control Conference, Seville, Spain, Dec. 2005, pp. 3553-3559.

[34] D. C. Conner, H. Choset, and A. A. Rizzi, "Towards provable navigation and control of nonholonomically constrained convex-bodied systems," in IEEE International Conference on Robotics and Automation, 2006, pp. 4416-4418.

[35] T. Liddy, T.-F. Lu, P. Lozo, and D. Harvey, "Obstacle avoidance using complex vector fields," in Proc. of the 2008 Australasian Conference on Robotics and Automation, Canberra, Australia, Dec. 2008.

[36] J. H. Ginsberg, Advanced Engineering Dynamics - 2nd Edition. Cambridge University Press, 1995.

[37] D. J. Griffiths, Introduction to Electrodynamics. Third Edition. Prentice Hall, Upper Saddle River, New Jersey, 1999.

[38] D. A. de Wolf, Essentials of Electromagnetics for Engineering. Cambridge University Press, 2001.

[39] S. M. LaValle, Planning Algorithms. Cambridge University Press, 2006. 